\title{
Idosos em cuidados paliativos: a vivência da espiritualidade frente à terminalidade
}

\author{
Older adults in palliative care: experiencing spirituality in the face of terminality
}

Adultos mayores en cuidados paliativos: la experiencia de la espiritualidad ante la terminalidad

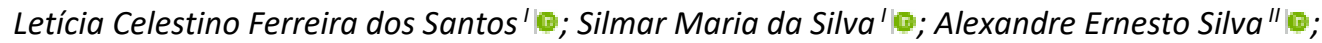 \\ Isabel Yovana Quispe Mendoza' $\mid \oplus$; Fabiano Moraes Pereira' ${ }^{\prime}$; Rosimeire Angela de Queiroz Soares ${ }^{\prime \prime \prime} \mid \oplus$
}

'Universidade Federal de Minas Gerais, Belo Horizonte, MG, Brasil; "Universidade Federal de São João Del Rei, São João Del Rei, MG, Brasil; "I'Faculdade de Ciências Médicas da Santa Casa de São Paulo, São Paulo, SP, Brasil

\begin{abstract}
RESUMO
Objetivo: compreender a vivência da espiritualidade de idosos em cuidados paliativos de um hospital público de Belo Horizonte. Método: trata-se de uma pesquisa qualitativa com 11 idosos em cuidados paliativos, por meio uma entrevista a partir de três questões norteadoras. Para análise dos dados, foram aplicadas as três etapas previstas no processo de análise de conteúdo. Resultados: emergiram duas categorias, "A espiritualidade no idoso em cuidados paliativos" e "A percepção do idoso sobre a abordagem da espiritualidade pela equipe de saúde". Conclusão: os idosos em cuidados paliativos vivenciam a espiritualidade, as relações com o transcendente, independentemente de possuir uma religião. E buscam adaptações às novas condições de vida, dando alívio dos sintomas por meio da relação com o sagrado.

Descritores: Idoso; Cuidados paliativos; Cuidados paliativos na Terminalidade da Vida; Espiritualidade.
\end{abstract}

\section{ABSTRACT}

Objective: to understand the experience of spirituality in older adults in palliative care at a public hospital in Belo Horizonte. Method: this qualitative study of 11 older adults in palliative care was conducted by interview based on three guiding questions. Data were processed by three-step content analysis. Results: two categories emerged, "Spirituality in older adults in palliative care" and "Older adults' perceptions of the health team's approach to spirituality". Conclusion: the older adults in palliative care experienced spirituality, relationships with transcendence, regardless of whether or not they had a religion. They endeavor to adapt to the new conditions of life and relieve their symptoms through relationships with things sacred.

Descriptors: Aged; Palliative care; Hospice Care; Spirituality.

\section{RESUMEN}

Objetivo: comprender la experiencia de la espiritualidad en adultos mayores en cuidados paliativos en un hospital público de Belo Horizonte. Método: este estudio cualitativo de 11 adultos mayores en cuidados paliativos se realizó mediante entrevista basada en tres preguntas orientadoras. Los datos se procesaron mediante análisis de contenido de tres pasos. Resultados: surgieron dos categorías, "Espiritualidad en adultos mayores en cuidados paliativos" y "Percepciones de los adultos mayores sobre el enfoque de espiritualidad del equipo de salud". Conclusión: los adultos mayores en cuidados paliativos experimentaron espiritualidad, relaciones con trascendencia, independientemente de si tenían religión o no. Se esfuerzan por adaptarse a las nuevas condiciones de vida y aliviar sus síntomas mediante relaciones con cosas sagradas.

Descriptores: Anciano; Cuidados paliativos; Cuidados paliativos ao Final de la Vida; Espiritualidad.

\section{INTRODUÇÃO}

Quando as terapias disponíveis já não oferecem resultados satisfatórios para aumentar a taxa de sobrevida, recomenda-se a adoção de uma assistência pautada nos Cuidados Paliativos (CP), compreendida como uma abordagem que objetiva a promoção da qualidade de vida do paciente e seus familiares, frente a doenças que ameaçam a continuidade da vida, por meio da prevenção e alívio do sofrimento, da dor e demais problemas de natureza física, psicossocial e espiritual, uma vez que estas podem gerar sofrimento físico, social, psicológico e/ou espiritual ${ }^{1}$.

Visto que a dimensão espiritual não pode ser dissociada do ser, a espiritualidade/religião constitui o quinto domínio de cuidado, dentre os oito domínios essenciais estabelecidos pelas diretrizes de prática clínica para a qualidade em $C P^{2}$.

Logo, os CP transpassam a dimensão da condição física, entremeando-se com os aspectos imateriais da espiritualidade ${ }^{1}$, pois considera que este domínio essencial contribui para o alívio da dor e do sofrimento face ao adoecimento e finitude ${ }^{3}$, a fim de uma melhor qualidade de vida possível a ser promovida ${ }^{4}$ ao paciente idoso em CP.

Partindo do pressuposto que a espiritualidade no paciente idoso em CP ajuda a superar os medos, auxilia o paciente a encontrar um sentido para a vida, superando obstáculos e limitações impostas pelo agravo de doença que ameacem a continuidade da vida, e que o cuidado deve perpassar pela integralidade do ser, questiona-se: Como é a vivência da espiritualidade, no contexto hospitalar, de idosos em cuidados paliativos? 
Compreender essa vivência pode evidenciar as nuances relacionadas ao cuidado prestado e fomentar reflexões acerca da espiritualidade na assistência ao paciente idoso em CP, com vistas a uma prática integral no ambiente hospitalar.

Assim, o presente estudo teve como objetivo compreender a vivência da espiritualidade de idosos em cuidados paliativos de um hospital público de Belo Horizonte.

\section{REFERENCIAL TEÓRICO}

O cuidado, de acordo com a Teoria do Cuidado Transpessoal de Jane Watson, é uma ciência humana desenvolvida a partir de fundamentos filosóficos e sistemas de valores, e compreende que o ser humano é um todo biológico, social e espiritual, que não pode ser fragmentado ${ }^{5}$. O cuidado envolve transcendência espiritual, intersubjetividade e dignidade humana. Seu foco está em proteger, amparar, preservar a dignidade humana, a integralidade do ser, a humanização da assistência para um eixo mais altruísta, espiritual fundamentado nas bases do conhecimento científico ${ }^{6}$.

Nesse sentido, o cuidado transpessoal visa se sobrepor à valorização da tecnologia, que estima somente a cura, e procura considerar como prioridade o próprio paciente, em todas as suas dimensões físicas, inclusive a espiritualidade ${ }^{1}$.

A espiritualidade pode ser entendida como universal e manifesta-se a partir do íntimo do indivíduo. Está relacionada a valores de harmonia e completude, conexão com o outro, interesse pelo próximo e por si, em unidade com a vida, com o universo, e com fim da vida terrena. É uma busca pessoal para compreender o sentido da vida, a relação com o sagrado ou transcendente, que pode ou não levar ao desenvolvimento de práticas religiosas ou formações de comunidades religiosas ${ }^{7}$.

Em CP as preocupações espirituais são um elemento comum da experiência da doença, e a espiritualidade pode influenciar na capacidade de lidar com doenças que não tem possibilidade de cura ${ }^{8}$, uma vez que eles encontram em suas crenças espirituais maneiras que ajudam a entender o sofrimento, a agonia e a incerteza de suas vida ${ }^{9}$.

\section{MÉTOdo}

Trata-se de uma pesquisa qualitativa ${ }^{10}$, realizada no Hospital das Clínicas da Universidade Federal de Minas Gerais, em Belo Horizonte, com 11 pacientes internados que atenderam os critérios de elegibilidade: idade de 60 anos ou mais, em acompanhamento com a equipe de CP, independentemente do tempo de acompanhamento, e que tinham compreensão da situação de saúde no qual se encontravam. Foram excluídos os que apresentavam registro no prontuário de alterações na cognição, incapacidade de se comunicar verbalmente, e os que não tinham conhecimento, a pedido da família ou acompanhantes, que estavam sendo acompanhados pela equipe de CP.

A coleta de dados ocorreu entre junho e julho de 2018, na própria instituição hospitalar, em sala privada, designada para o paciente que conseguia deambular. Para os pacientes impossibilitados de caminhar e/ou sair do leito, as entrevistas foram realizadas a beira leito, sendo utilizados biombos para preservar a privacidade. Foram utilizadas três questões norteadoras ${ }^{10}$ : A fé é algo importante para você? Conte mais sobre isso; Como você acredita que sua fé ajudaria nesse momento de sua vida?; Como você gostaria que a equipe de saúde abordasse a "fé" em seu atendimento?

Optou-se pelo termo fé no lugar de espiritualidade, pois na primeira entrevista percebeu-se uma dificuldade do participante em compreender o termo espiritualidade. Contudo, o entendimento foi facilitado ao substituir por fé, sem prejuízo no prosseguimento da entrevista.

As entrevistas foram gravadas em gravador digital e transcritos os discursos, respeitando a sequência das ideias, a linguagem, as pausas e as repetições realizadas. Após, foram aplicadas as três etapas previstas no processo de análise de conteúdo de Bardin: pré-análise, exploração do material e tratamento dos resultados ${ }^{11}$.

Na pré-análise foi realizada leitura e releitura dos discursos, na exploração do material foi realizado o agrupamento dos trechos das entrevistas que apresentavam ideias em comum, o que permitiu a codificação e posterior categorização para a descrição analítica. Em seguida, passou-se para a interpretação inferencial, com a separação dos conteúdos com sentidos semelhantes a partir de recortes das falas, formando assim as duas categorias: "A espiritualidade no idoso em cuidados paliativos" e "A percepção do idoso sobre a abordagem da espiritualidade pela equipe de saúde".

A fim de preservar o anonimato, cada participante foi identificado com a letra $E$, de entrevistado, seguida pelo número de ordem da entrevista.

O estudo foi submetido e aprovado pelo Comitê de Ética em Pesquisa da Universidade Federal de Minas Gerias (parecer $n$ ㅇ 2.650.177) e foram aplicados aos participantes o Termo de Consentimento Livre e Esclarecido, em duas vias, em conformidade com a Resolução no 466/2012 do Conselho Nacional de Saúde. 


\section{RESULTADOS E DISCUSSÃo}

Participaram do estudo 11 idosos em CP, sendo a maioria (72,7\%) do sexo masculino, entre 60 a 70 anos (63,6\%), casado(a)/união estável (54,4\%), com ensino fundamental (72,7\%) e procedentes do interior do estado de Minas Gerais (54,5\%). A maioria estava sendo acompanhada pela equipe de CP há menos de um mês, sendo 4 (45,4\%) há menos de uma semana e $3(27,2 \%)$ entre duas a quatro semanas.

\section{A espiritualidade no idoso em cuidados paliativos}

O entendimento da espiritualidade:

A fé para mim representa esperança... e tem uma frase que diz assim: mais importante que viver é aquilo que dá razão a vida... (E3)

Ahhh, a fé me dá mais segurança! A fé é meu escudo (E2)

A fé desencadeia a contemplação e a reflexão das experiências existenciais, além de nortear a busca do sentido da vida ${ }^{7}$. Esta dimensão do significado da espiritualidade é um fenômeno intimamente humano, que não se adapta em uma completa definição. É difícil encontrar um consenso na elaboração deste conceito ${ }^{12}$.

Por vezes, a fé para o idoso em CP está relacionada com Deus:

Se a pessoa não tiver fé em Deus, ela não tem fé em nada né... (E7)

Se não fosse Deus eu não estava mais aqui não... todo dia de manhã eu oro, peço a Deus para me ajudar... (E8)

A fé pode ser compreendida como ato de crer na existência de algo superior, dispensando comprovações materiais. E Deus pode representar este ser superior, uma força maior que rege o universo e a vida de cada pessoa ${ }^{13}$.

A espiritualidade e religiosidade estão presentes no dia-a-dia dos pacientes idosos com doença ameaçadora da vida, se constituem em estratégias utilizadas para enfrentar os desafios, desconfortos, sofrimentos e incertezas do processo de adoecimento; recorrem a Deus e às suas crenças em momentos de aflição e desespero. A busca pelo sagrado ocorre diariamente diante do vazio existencial instalado ${ }^{14}$ :

Eu entendo a minha doença...eu não me curvo diante da doença também.... eu busco forças no imprevisível, no impossivel, porque eu já vi ele acontecer... (E4)

Face à finitude, podem emergir as incertezas, principalmente, quando há dificuldades em encontrar respostas para as novas demandas. Esta situação pode pôr em risco a relação do idoso com o transcendente e a busca pela esperança.

Eu frequentava a Igreja [...] sabe, mas aí, eu até conversei com rapaz ali hoje e eu tô até esmorecido com a doutrinação deles lá, sabe? Tô começando a procurar uma outra coisa que me toque mais... (E3)

Diante da notícia de um prognóstico ruim, o idoso sugere sutilmente a ideia de negociação com Deus, na busca de estabelecer um acordo e obter ou ampliar a busca pela esperança.

Apesar dos pacientes reagirem de diferentes formas à morte, todos vivenciam o luto antecipatório, um processo composto por cinco fases: 1) negação e isolamento, 2) raiva, 3) negociação/barganha, 4) depressão e 5) aceitação ${ }^{15}$.

A espiritualidade auxilia na condução à aceitação:

Vocês estudam, analisam, fazem isso, faz aquilo, mas quem manda é Ele lá em cima... é Ele quem vai determinar, não vai ser o médico....... Aí não tem como eu reclamar alguma coisa, só viver e aceitar isso bem, sem sofrer e chorar (E1)

Nas situações em que as doenças não têm possibilidade de cura, a dimensão espiritual possibilita aos pacientes o desenvolvimento da esperança, de um significado para a doença e de um propósito e sentido para a vida, o que favorece o amadurecimento pessoal, a integridade e o enfrentamento da situação vivenciada ${ }^{16}$.

A espiritualidade e a religiosidade são recursos capazes de proporcionar alívio nos momentos difíceis e ajudam a buscar o bem-estar e ressignificação do sentido da vida, diante daquilo que não pode ser modificado ${ }^{14}$ :

"Eu oro antes de sair pra pedir a Deus para melhorar as dores que sinto...pensei que nunca mais iria andar...E Deus me ajudou.... E como não tem cura, e só vai piorando, Deus é o melhor remédio, peço a Deus tudo e a dor passa!!" (E9)

"A fé que eu tenho é que eu vou andar! E Deus está no poder! E a fé que eu tenho, de acabar todas essas dores no meu corpo, porque eu mesmo na cadeira de rodas eu vou mexer! Se você põe na sua vida você vai vencer!" (E10)

A partir da espiritualidade, é atribuído sentido ao sofrimento, aliviando-o ${ }^{17}$. Logo, o ser humano sente-se estimulado a buscar sentido para vida, e a vida tem sentido como um todo, e o sofrimento inevitável tem igualmente seu sentido e faz parte da vida ${ }^{18}$. 
Praticar a espiritualidade diante das situações que promulguem a finitude do ser humano torna-se essencial para o seguimento da vida e a instalação da esperança dos idosos em CP, sendo essa prática considerada essencial para responder aos ensejos desses indivíduos quanto a sua própria existência. Pois a esperança é um sentimento que estimula o ser humano no seu existir, permitindo um futuro otimista. É acreditar que algo é possível mesmo quando há fortes evidências do contrário ${ }^{13}$.

Os profissionais de enfermagem que compreendem o momento vivido pelos pacientes e familiares, favorecem a construção de um sentido em meio à dor e o medo, aliviam a culpa, recuperam a esperança, relembram o paciente dos seus sonhos e desejos e oferecem à família acolhimento em seu sofrimento ${ }^{19}$.

\section{A percepção do idoso sobre a abordagem da espiritualidade pela equipe de saúde}

Kübler-Ross ${ }^{20}$ compartilha que, quando começou a abordar assuntos como transcendência, plenitude e vida após a morte com os pacientes, foi alvo de inúmeras críticas negativas pela própria equipe de saúde, pois para muitos a morte e o contato com forças superiores eram assuntos que nunca deveriam ser tocados, e a espiritualidade era vista como algo que somente igrejas, padres e doutrinadores poderiam retratar ${ }^{20}$.

Alguns idosos expressaram esse entendimento acerca da abordagem da espiritualidade pela equipe de saúde:

Chegar alguém aqui e perguntar como eu tô, e vem falando que sabe que Deus te ama né... eu não quero isso não, entendeu?... (E1)

A bíblia explica pra gente... não gente de hospital... Porque a salvação não vem do homem, vem só de Deus. Então alguém aqui no hospital falar disso, não mudaria em nada para mim... (E5)

Confundir o significado de espiritualidade com religiosidade pode prejudicar nas intervenções direcionadas ao cuidado espiritual, uma vez que a religião é apenas uma dimensão da espiritualidade, e abordar aspectos espirituais melhora a aceitação dos pacientes idosos em CP no processo de finitude da vida ${ }^{21}$.

Alguns entrevistados disseram preferir conversar sobre espiritualidade com pessoas mais próximas:

Eu já tô tenho a minha cunhada né... tá vindo aqui ler a bíblia... então tá bom assim... eu gosto que seja pessoas mais próximas a mim, para falar disso... aí eu me sentiria melhor...(E6)

Cada tem uma fé diferente né... Eu acho que não faria diferença na minha vida se um profissional de saúde viesse aqui falar de fé...mas prefiro conversar disso com minha esposa... (E11)

A discussão sobre espiritualidade exige habilidades avançadas de comunicação. Esta torna-se essencial para a adequada relação entre profissional de saúde, paciente e família. É ferramenta vital no campo da saúde, pois fortalece relações, amplia a autonomia do paciente e estreita o vínculo de confiança. No entanto, comunicar não é um ato simples; envolve crenças e valores singulares que podem interferir na transmissão das informações ${ }^{22}$.

Watson 6 relaciona o "ser cuidado" com o "ser cuidador" indo além de realizações de técnicas e cumprimento de tarefas, focando em desejos, emoções, indo ao encontro da essência do outro, respeitando sua cultura, origem e outras situações que possam interferir no processo do cuidado ${ }^{6}$.

Assim, para facilitar a abordagem da espiritualidade com o paciente, deve-se evitar utilizar regras rígidas e inflexíveis, o que pode aumentar a complexidade da dimensão espiritual ou remeter somente a ideia de religião. Incluir termos religiosos e não religiosos, traçar ações que sejam definidas pelo próprio paciente podem facilitar este processo. É importante que o maior número de pacientes tenha oportunidade de ter suas necessidades espirituais identificadas, sem importar da forma como eles a entendam.

A construção da comunicação terapêutica depende do grau de comprometimento do paciente. O profissional pode iniciar a comunicação passando informações que confortam, esclarecem e dignificam a finitude humana. Posteriormente, a relação interpessoal que acontece entre o profissional-paciente-familiar depende das habilidades de comunicação ${ }^{23}$.

Mas, por vezes, muitos profissionais, por estarem com excesso de demandas gerenciais e pelo quantitativo elevado de pacientes que acompanham, não conseguem estabelecer uma comunicação adequada, porque não dão espaço para o paciente falar sobre suas questões ${ }^{24}$. Em muitos casos o profissional de saúde, no primeiro encontro com o paciente aborda, precocemente, questões existenciais e espirituais, gerando sentimentos de exposição e invasão da privacidade no paciente.

Para o profissional de saúde que trabalha no campo dos cuidados paliativos, faz-se necessário priorizar as relações interpessoais de pacientes frente a finitude e de seus familiares, conhecendo expectativas, desejos, medos e anseios. Por meio da escuta e do diálogo é possível conduzir cada caso, promovendo o acolhimento embasado na compaixão ${ }^{6}$. 


\section{CONCLUSÃO}

Os idosos em CP vivenciam a espiritualidade, as relações com o transcendente, independentemente de possuir uma religião. E buscam adaptações às novas condições de vida, dando alívio dos sintomas por meio da relação com o sagrado. A espiritualidade, por vezes, foi a fonte de esperança para o enfrentamento das fases do luto antecipatório e força motriz para superar as notícias de um prognóstico ruim.

Embora reconhecessem os benefícios da espiritualidade, manifestaram não serem favoráveis à abordagem da espiritualidade pela equipe de saúde. Com vistas a uma prática integral no ambiente hospitalar, esse achado cabe uma reflexão das equipes de saúde com vista a um cuidado humanizado, integral, holístico, abarcando também a dimensão espiritual dos pacientes.

Frente a isso, e diante da limitação de estudos específicos para o idoso que vivenciam a espiritualidade diante a terminalidade, é possível identificar a necessidade de novos estudos a fim de subsidiar ações que auxiliem os profissionais de saúde a prestarem um cuidado idoso em CP baseada no amor, compaixão e dignidade humana.

Em relação a limitação da pesquisa apresentada, por ser tratar de um estudo qualitativo, não se pode generalizar os resultados encontrados em uma amostra, para uma população.

\section{REFERÊNCIAS}

1. Worl Health Organization. WHO definition of palliative care. [Internet], Genebra; 2002. [cited 2020 Mar 10] Available from: http://www.who.int/cancer/palliative/definition/en/

2. National Coalition for Hospice and Palliative Care. Clinical practice guidelines for quality palliative care. 4 a ed. [Internet], Richmond; 2018. [cited 2020 Mar 10] Available from: https://www.nationalcoalitionhpc.org/wpcontent/uploads/2018/10/NCHPC-NCPGuidelines_4thED_web_FINAL.pdf

3. Meneguin S, Matos TDS, Ferreira MLSM. Perception of cancer patients in palliative care about quality of life. Rev. Bras. Enferm. [Internet]. 2018 [cited 2020 Aug 20]; 71(4):1998-2004. DOI: http://dx.doi.org/10.1590/0034-7167-2017-0360

4. Borsatto AZ, Santos ADS, Progianti JM, Vargens OMC. Medicalization of death and palliative care. Rev. enferm. UERJ [Internet]. 2019 [cited 2020 Aug 20]; 27:e41021 DOI: http://dx.doi.org/10.12957/reuerj.2019.41021

5. Savieto RM, Leão ER. Nursing assistance and Jean Watson: a reflection on empathy. Esc. Anna Nery [Internet]. 2016 [cited 2020 Aug 20]; 20(1): 198-202. DOI: https://www.scielo.br/scielo.php?pid=S1414-81452016000100198\&script=sci_arttext\&tlng=en

6. Silva AE. A produção de cuidados paliativos no contexto da atenção domiciliar. [tese de Doutorado]. Belo Horizonte: Universidade Federal de Minas Gerais; 2018.

7. Balducci L. Geriatric oncology, spirituality, and palliative care. J. Pain Symptom Manage [Internet], 2018 [cited 2020 Mar 09]; 57(1):171-5. DOI: https://doi.org/10.1016/j.jpainsymman.2018.05.009

8. Cuartas-Hoyos P, Charry-Hernández R, Ospina-Muñoz P, Carreño-Corredor S. Spiritual care: a look from the perspective of the model of symptom management and palliative care. Rev. Colomb. Enferm. [Internet], 2019 [cited 2020 Mar 08]; 18(1):1-17. DOI. https://doi.org/10.18270/rce.v18i1.2341

9. Academia Nacional de Cuidados Paliativos. Manual de Cuidados Paliativos. [Internet]. São Paulo; 2012. 2a ed. [cited 2020 Mar 08] Available from: https://paliativo.org.br/download/manual-de-cuidados-paliativos-ancp/

10. Polit DF, Beck CT. Fundamentos de Pesquisa em Enfermagem. 9.ed. Porto Alegre: Artmed, 2018.

11. Bardin L. Análise de conteúdo. Tradução de Luis Antero Reto e Augusto Pinheiro. São Paulo: Edições 70/Livraria Martins Fontes; 2011.

12. Nunes MGS, Leal MCC, Marques APO, Mendonça SS. Long-lived elderly: assessment of quality of life in the field of spirituality, religiousness and personal beliefs. Saúde Debate [Internet], 2017 [cited 2020 Feb 25]; 41:1102-15. DOI: https://doi.org/10.1590/0103-1104201711509.

13. Borges MS, Santos MBC, Pinheiro TG. Social representations about religion and spirituality. Rev. bras. enferm. [Internet], 2015 [cited 2020 Feb 28]; 68(4):609-16. DOI: https://doi.org/10.1590/0034-7167.2015680406i

14. Freitas RA, Menezes TMO, Santos LB, Moura HCGB, Sales MG, Moreira FA. Spirituality and religiousity in the experience of suffering, guilt, and death of the elderly with câncer. Rev Bras Enferm. [Internet]. 2020 [cited 2020 Aug 19]; 2020; 73(Suppl 3):e2019003. DOI: https://doi.org/10.1590/0034-7167-2019-0034

15. Kubler-Ross E. Sobre a morte e o morrer. 10a ed. São Paulo: Martins Fontes; 2017.

16. Benites AC, Neme CMB, Santos MA. Significance of spirituality for patients with cancer receiving palliative care. Estud. Psicol. [Internet], 2017 [cited 2020 Feb 28]; 34(2):269-79. DOI: https://doi.org/10.1590/1982-02752017000200008

17. Arrieira ICO, Thofehrn MB, Milbrath VM, Schwonke CRGB, Cardoso DH, Fripp JC. The meaning of spirituality in the transience of life. Esc. Anna Nery 2017 [cited 2020 Aug 19]; 21(1):e20170012. Available from: https://www.scielo.br/scielo.php?pid=S1414$81452017000100212 \&$ script=sci_arttext\&tIng=en

18. Arrieira ICO, Thofehrn MB, Porto AR, Moura PMM, Martins CL, Jacondino MB. Spirituality in palliative care: experiences of an interdisciplinary team. Rev. Esc. Enferm. USP. [Internet]; 2018 [cited 2020 Feb 28]; 52:e03312. DOI: https://doi.org/10.1590/s1980-220x2017007403312.

19. Góes MGO, Crossetti MGO. Developing a spiritual care model for patients and their relatives in illness. Rev. Gaúcha Enferm. [Internet]. 2020 [cited 2020 Aug 19]; 41(esp)e20190150. DOI: https://doi.org/10.1590/1983-1447.2020.20190150 
20. Kubler-Ross E. A roda da vida. [Internet]. Rio de Janeiro: Sextante; 1998. [cited 2020 Mar 10] Available from: http://www.espiritualidades.com.br/Artigos/K_autores/Kluber-Ross_Elisabeth_tit_Roda_da_vida-A.pdf.PDF

21. Matos JC, Guimarães SMF. The application of transpersonal and spiritual care for older adults receiving palliative care. Rev. bras. geriatr. gerontol. [Internet]. 2020 [cited 2020 Aug 19]; 22(5):e190186. DOI: http://dx.doi.org/10.1590/198122562019022.190186

22. Koch CL, Rosa AB, Bedin SC. Bad news: meanings attributed in neonatal/pediatric care practices. Rev. Bioét. [Internet]. 2017 [cited 2020 Mar 08]; 25(3): 577-84. DOI: https://doi.org/10.1590/1983-80422017253214

23. Barbosa RMM, Ferreira JLP, Melo MCB, Costa JM. A Spirituality as a coping strategy for families of adult patients in palliative care. Rev. SBPH [Internet]. 2017 [cited 2020 Mar 08]; 20(1):165-82. Available from: http://pepsic.bvsalud.org/scielo.php?script=sci_arttext\&pid=S1516-08582017000100010

24. Meneguin S, Banja PFT, Ferreira MLS. Care for hospitalized elderly patients: implications for nursing team. Rev. enferm. UERJ [Internet]. 2017 [cited 2020 Mar 08]; 25:e16107. DOI: https://doi.org/10.12957/reuerj.2017.16107 